\title{
Accretion-powered millisecond pulsars
}

\author{
J. Poutanen \\ Astronomy Division, P.O.Box 3000, FIN-90014 University of Oulu, Finland
}

\begin{abstract}
I review X-ray observations of accretion-powered millisecond pulsars and current theories for formation of their spectra and pulse profiles.
\end{abstract}

Key words: accretion, accretion discs, stars: neutron, X-rays: binaries PACS: 95.75.Wx, 95.85.Nv, 97.10.Gz, 97.60.Gb, 97.60.Jd, 97.80.Jp

\section{Introduction}

A number of rapidly spinning neutron stars in low-mass X-ray binaries were discovered with the Rossi X-ray Timing Explorer ( $R X T E)$ in the recent years. These discoveries confirm the ideas on the formation of radio (recycled) millisecond pulsars in low-mass X-ray binaries (see review in e.g. [2]). Accretion of matter onto a neutron star results in its spin increase to millisecond periods if the magnetic field of the star is below about $10^{9} \mathrm{G}$.

Thirteen sources show nearly coherent oscillations for a few seconds during X-ray bursts at frequencies ranging from 270 to $619 \mathrm{~Hz}$ (see [51] for a review). These are now called nuclear-powered millisecond pulsars. The number of accretion-powered millisecond pulsar (AMSP) showing pulsations in the persistent emission reached seven by June 2005. A more observationally inclined review of AMSP is given in [56]. Here I concentrate on the results of the X-ray spectroscopy, analysis of pulse profiles, and our present theoretical understanding.

\section{Pulsars parameters}

The first real AMSP SAX J1808.4-3658 was discovered in 1998 by Wijnands $\&$ van der Klis [57]. Now (September 2005) there are seven AMSPs with 
Table 1

Parameters of the accretion-powered millisecond pulsars

\begin{tabular}{llccccc}
\hline Source & $\begin{array}{c}P_{\text {orb }}{ }^{a} \\
(\min )\end{array}$ & $\begin{array}{c}\nu_{\text {spin }}{ }^{b} \\
(\mathrm{~Hz})\end{array}$ & $\begin{array}{c}a_{x} \sin i^{c} \\
(\text { lt-ms })\end{array}$ & $\begin{array}{c}f_{x}{ }^{d} \\
\left(\mathrm{M}_{\odot}\right)\end{array}$ & $\begin{array}{c}M_{\mathrm{c}, \min }{ }^{e} \\
\left(\mathrm{M}_{\odot}\right)\end{array}$ \\
\hline 1 & SAX J1808.4-3658 & 121 & 401 & 62.809 & $3.779 \times 10^{-5}$ & 0.043 \\
2 & XTE J1751-305 & 42.4 & 435 & 10.113 & $1.278 \times 10^{-6}$ & 0.014 \\
3 & XTE J0929-314 & 43.6 & 185 & 6.290 & $2.9 \times 10^{-7}$ & 0.0083 \\
4 & XTE J1807-294 & 40.1 & 191 & 4.75 & $1.49 \times 10^{-7}$ & 0.0066 \\
5 & XTE J1814-338 & 257 & 314 & 390.3 & $2.016 \times 10^{-3}$ & 0.17 \\
6 & IGR J00291+5934 & 147 & 599 & 64.993 & $2.813 \times 10^{-5}$ & 0.039 \\
7 & HETE J1900.1-2455 & 83.3 & 377 & 18.39 & $2.00 \times 10^{-6}$ & 0.016 \\
\hline
\end{tabular}

${ }^{a}$ Orbital period; ${ }^{b}$ neutron star spin frequency; ${ }^{c}$ projected semimajor axis; ${ }^{d}$ pulsar mass function; ${ }^{e}$ minimum companion mass (for a $M=1.4 \mathrm{M}_{\odot}$ neutron star).

References: (1) [57,7]; (2) [27,29]; (3) [47,14]; (4) [30,21,12]; (5) [28,31]; (6) $[11,33,32,15] ;(7)[53,37,20]$.

spin frequencies from $185 \mathrm{~Hz}$ up to $599 \mathrm{~Hz}$ (see Table 1). The fastest AMSP, IGR J00291+5934 with the period of just $1.67 \mathrm{~ms}$ is the fifth fastest among all known pulsars (including radio- and nuclear-powered MSPs). The last AMSP HETE J1900.1-2455 was discovered in June 2005.

AMSPs show pulse frequency variations. These observations are very important for understanding of the evolution of the neutron stars in low-mass X-ray binaries towards radio MSPs. They would also shed some light on a complicated problem of the interaction of the magnetosphere with the accretion flow. One expects a spin-up rate

$$
\dot{\nu}=3.7 \times 10^{-13} \frac{L_{37}}{\eta_{-1} I_{45}}\left(\frac{R_{\mathrm{m}}}{R_{\mathrm{co}}}\right)^{1 / 2}\left(\frac{M}{1.4 \mathrm{M}_{\odot}}\right)^{2 / 3}\left(\frac{\nu_{\mathrm{spin}}}{600}\right)^{-1 / 3} \mathrm{~Hz} \mathrm{~s}^{-1},
$$

where (notation $Q=10^{x} Q_{x}$ in cgs units is used) $I$ is the neutron star moment of inertia, $L$ is the luminosity, $\eta$ is the accretion efficiency, $R_{\mathrm{m}}$ and $R_{\text {co }}$ are the magnetospheric and corotation radii. Some reported $\dot{\nu}$ are, however, negative implying pulsar slowing down during the outburst [14,36]. Markwardt [26] finds wild swings in the apparent spin frequency of both signs resulting in the total fractional phase shift less than 0.15 . These could be, however, artifacts of the pulse profile variations (see Sect. 4 ). The reported positive $\dot{\nu} \sim 8 \times 10^{-13}$ $\mathrm{Hz} / \mathrm{s}$ for IGR J00291+5934 [13] is larger than expected by a factor of 5 (since $\left.L_{37} \sim 0.37\right)$. A positional error of $\sim 0^{\prime \prime} .7$ could result in such a large derivative, while the radio position is known with a 0 "' 1 accuracy [50]. The motion of the radio source itself does not produce an error larger than $\sim 0$ "'.3 (for the 
distance of $5 \mathrm{kpc}$ ) a week after the outburst, confirming the reality of the pulsar spin-up and possibly implying a small moment of inertia $I$.

Accretion-powered pulsars reside in very compact binary systems with orbital periods ranging from $40 \mathrm{~min}$ to $4.3 \mathrm{~h}$. Surprisingly three out of 7 pulsars have $42 \pm 2 \mathrm{~min}$ orbits. Adding to this set also 4U1626-67, 4U1916-05 and X1832-330 (in globular cluster NGC 6652) with orbital periods of 42, 50, and $44 \mathrm{~min}$, respectively, it becomes clear that this interesting fact deserves some explanation (see [40] for a possible scenario). The pulsar mass function

$$
f_{x}=\left(M_{\mathrm{c}} \sin i\right)^{3} /\left(M_{\mathrm{c}}+M_{x}\right)^{2}=4 \pi^{2}\left(a_{x} \sin i\right)^{3} / G P_{\text {orb }}^{2}
$$

is very low for all the objects implying extremely low companion masses consistent with degenerate white (helium or carbon-oxygen) or brown dwarfs [4,29,14,12,15] except XTE J1814-338 which contains probably a hydrogenrich star [22]. All AMSPs are transients with the outbursts repeating every few years and lasting a few weeks. They have a rather low time-average accretion rate of $\sim 10^{-11} \mathrm{M}_{\odot} / \mathrm{yr}$, which could be the main reason for magnetic field still to be strong enough for pulsations to be observed [10].

\section{Broad-band X-ray spectra}

The broad-band coverage of the $R X T E$ together with $X M M$ gave a possibility to study the spectra of AMSP in great details. The spectra can be modelled by three components: two soft, thermally looking ones below a few keV and a power-law like tail (see Fig. 1).

The two soft components which can be modelled as thermal emission from a colder $(k T \sim 0.4-0.6 \mathrm{keV})$ accretion disc and a hotter $(\sim 1 \mathrm{keV})$ spot on the neutron star surface. The softer components in XTE J1751-305 and XTE J1807-294 are studied in [16,12] with the XMM. The obtained inner disk radius $R_{\text {in }} \sim 10-15 \mathrm{~km} / \sqrt{\cos i}$ is consistent with the flow disrupted by the neutron star magnetosphere within a couple of stellar radii. The hotter black body normalization corresponding to the area of $\sim 30-100 \mathrm{~km}^{2}$ and its pulsation are consistent with it being produces in a spot at the neutron star surface.

A power-law tail (having spectral photon index $\Gamma \sim 1.8-2.1$ ) shows a cutoff around $100 \mathrm{keV}$ and can be fitted with thermal Comptonization. The electron temperature of the Comptonizing medium is around $k T_{\mathrm{e}} \sim 20-60 \mathrm{keV}$ and Thomson optical depth of $\tau_{\mathrm{T}} \sim 0.7-2.5$ (for a plane-parallel slab geometry) $[17,45,16,12]$. Weakness of the Compton reflection from the disk indicates that the solid angle covered by the disk as viewed from the main emission source (accretion shock) is small, being consistent with $R_{\text {in }} \sim 40 \mathrm{~km}$ [16]. 


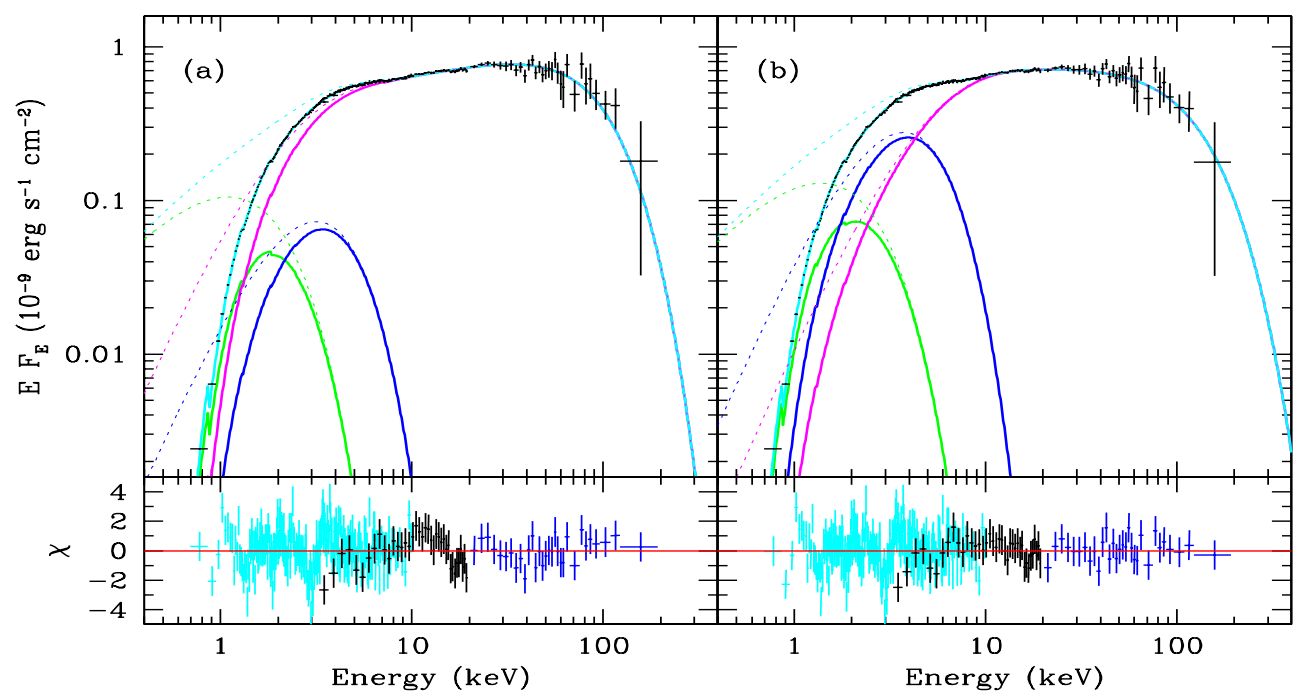

Fig. 1. Spectrum of XTE J1751-305 as observed by XMM/EPIC-pn, RXTE/PCA and HEXTE. The models consists of the multicolour disc (peaking at $\sim 1 \mathrm{keV}$ ), single-temperature blackbody (peaking at $3-4 \mathrm{keV}$ ), and thermal Comptonization of the blackbody photons. The dotted curves show unabsorbed spectral components. Panel (a) shows a model where seed photons for Comptonization have the same temperature as the blackbody photons, while in panel (b) the seed photons are hotter. The lower panels shows the residuals. See [16] for details.

The broad-band X-ray spectra of AMSPs are very similar to each other. They also show very little variability during the outbursts (see e.g. $[18,16,13])$. When fitting spectra with thermal Comptonization models, one also finds that the product of the electron temperature and optical depth is almost invariant (e.g. $\left[k T_{\mathrm{e}}, \tau_{\mathrm{T}}\right]=[60 \mathrm{keV}, 0.88]$ in SAX J1808.4-3658; [33 keV, 1.7] in XTE J1751305, [37 keV, 1.7] in XTE J1807-294, [49 keV, 1.12] in IGR J00291+5934; see $[16,12,13])$. The constancy of the spectral slope can be used as an argument that the emission region geometry does not vary much with the accretion rate. If the energy dissipation takes place in a hot shock, while the cooling of the electrons is determined by the reprocessing of the hard X-ray radiation at the neutron star surface (two-phase model, e.g. $[19,46,25]$ ), the spectral slope is determined by the energy balance in the hot phase and, therefore, by the geometry.

\section{Phase-resolved spectroscopy, pulse profiles, and time lags}

The pulse profiles from AMSPs are rather close to sinusoidal with peak-topeak oscillation amplitude $A=\left(F_{\max }-F_{\min }\right) /\left(F_{\max }+F_{\min }\right)$ between 4 (in XTE J1751-305) and 12 per cent (in XTE J1814-338). At energies above 50 $\mathrm{keV}$ the INTEGRAL data on IGR J00291+5934 show the increase of the pulse amplitude up to $\sim 25$ per cent [13]. Deviations from the sine wave are stronger 


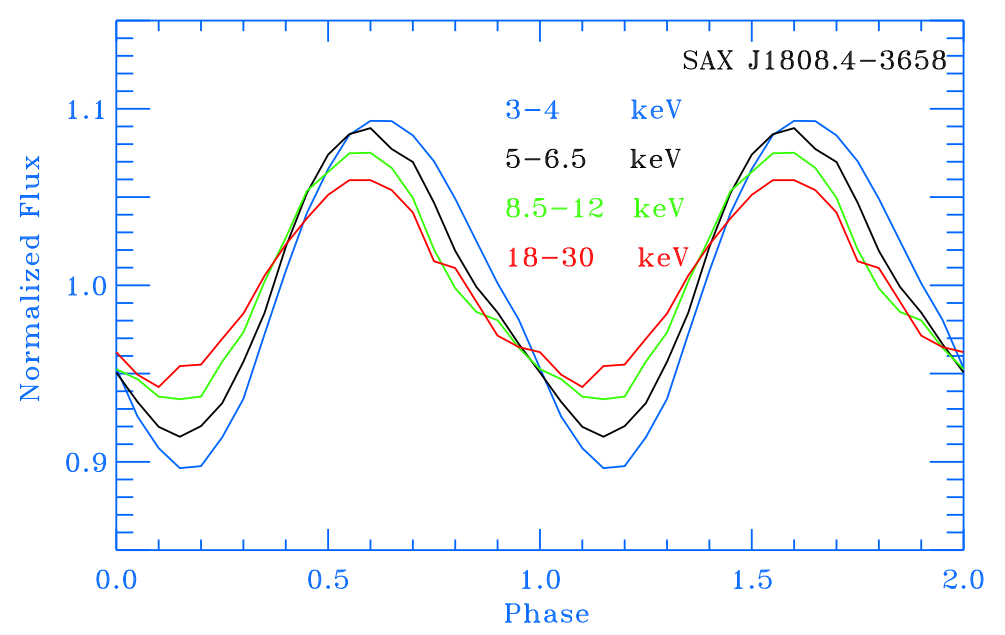

Fig. 2. Normalized pulse profiles of SAX J1808.4-3658 at different energies during the April 1998 outburst.
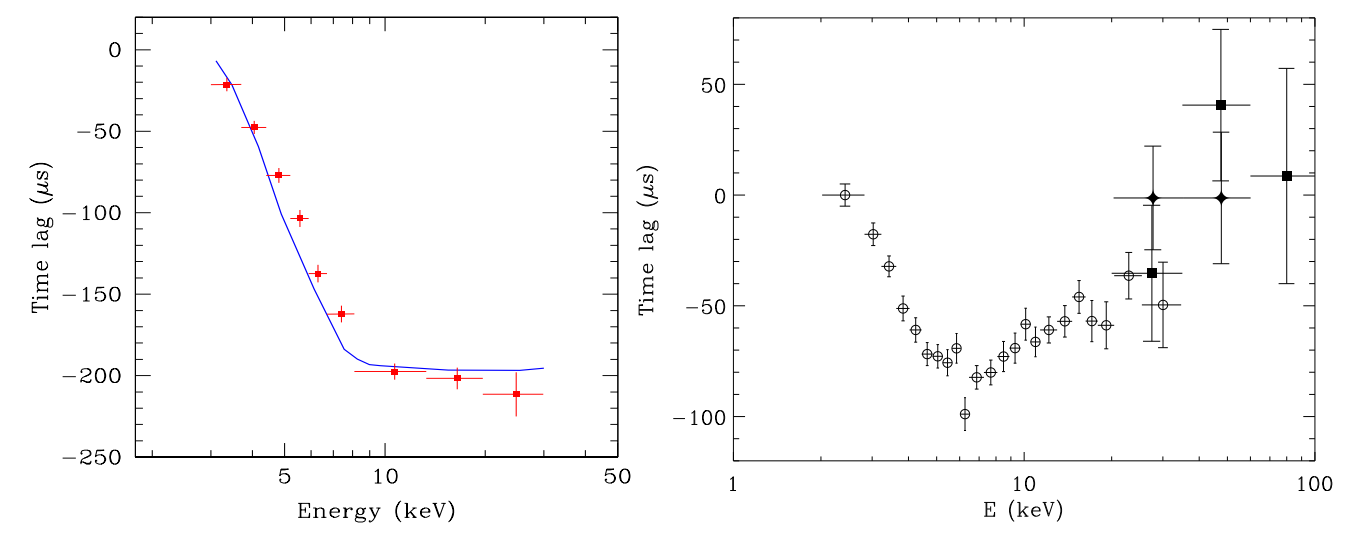

Fig. 3. Time lags as a function of energy for SAX J1808.4-3658 (left panel; [17]) and IGR J00291+5934 (right panel; [13]).

at higher energies (see Fig. 2). The harmonic content also is stronger when $A$ is larger (e.g. the harmonic-to-fundamental ratio is $\sim 0.03$ in XTE J1751-305 [16], while it is $\sim 0.33$ in XTE J1814-338 [52]).

Pulse profiles at higher energies reach their peaks at an earlier phase relative to the soft photons resulting in the soft time lags. In SAX J1808.4-3658 [9,17], XTE J1751-305 [16], and XTE J0929-314 [14] the lags increase (in absolute value) with energy up to about $7-10 \mathrm{keV}$ after they saturate (see Fig. 3, left panel). In IGR J00291+5934 (see Fig. 3, right panel; $[15,13]$ ), the behaviour is more complicated and the lags decrease between 7 and $15 \mathrm{keV}$, seemingly saturating at higher energies.

One can point out that the contribution of the black body decreases exponentially with energy and the lags increase significantly at the same time. Above $\sim 7 \mathrm{keV}$, the blackbody's contribution is negligible and the lags saturate. When fitting phase-resolved spectra with a two-component model (blackbody 
(a)

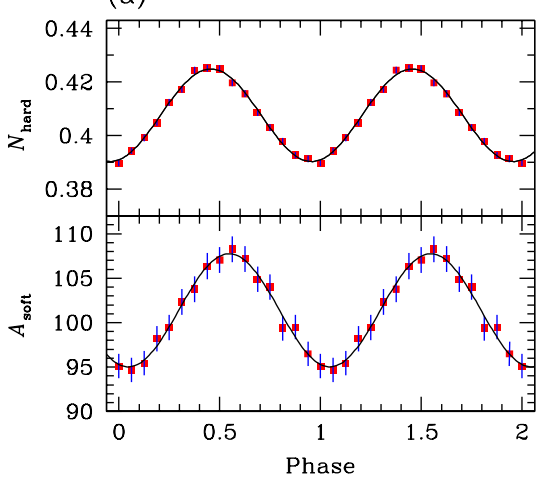

(b)

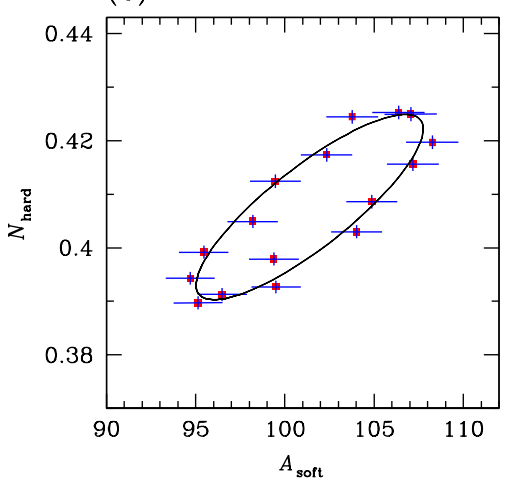

Fig. 4. Pulsation of the two model components, blackbody and Comptonization, in XTE J1751-305. From [16].

+ Comptonization), one clearly sees that the normalizations of the components do not vary in phase, with black body lagging the Comptonized emission (see Fig. 4 and $[17,16])$. In SAX J1808.4-3658 the profile corresponding to the Comptonized emission is clearly non-sinusoidal. Difference in profiles of the two components can be explained by their different emission pattern [45]. Its variation may cause pulse shape change resulting in the swings of $\dot{\nu}$ discussed in Sect. 2.

\section{$5 \quad$ Modelling the pulse profiles of AMSPs}

One believes that the bulk of the X-ray emission observed from AMSPs originates from polar caps where the gas stream channels by the neutron star magnetic field impacts the stellar surface forming a shock. This is supported by a weak energy dependence of the variability amplitude and a fairly constant spectral shape as a function of pulse phase (any additional source of radiation would have to have a spectrum identical to that of the shock), as well as the observed broadening of the pulse peak in the power-spectrum of SAX J1808.4-3658 due to modulation of the aperiodic variability by the spin period [34].

\subsection{Computing pulse profiles}

Pulse profile shape and variability amplitude carry the information about the compactness of the neutron star, spot size and its position at the star, and the emission pattern. Gravitational light bending tends to decrease the pulse amplitude $[42,48,41,24,1]$. An earlier attempt to incorporate the effect of rapid rotation using Kerr metric [8] did not include the dominant Doppler boost 


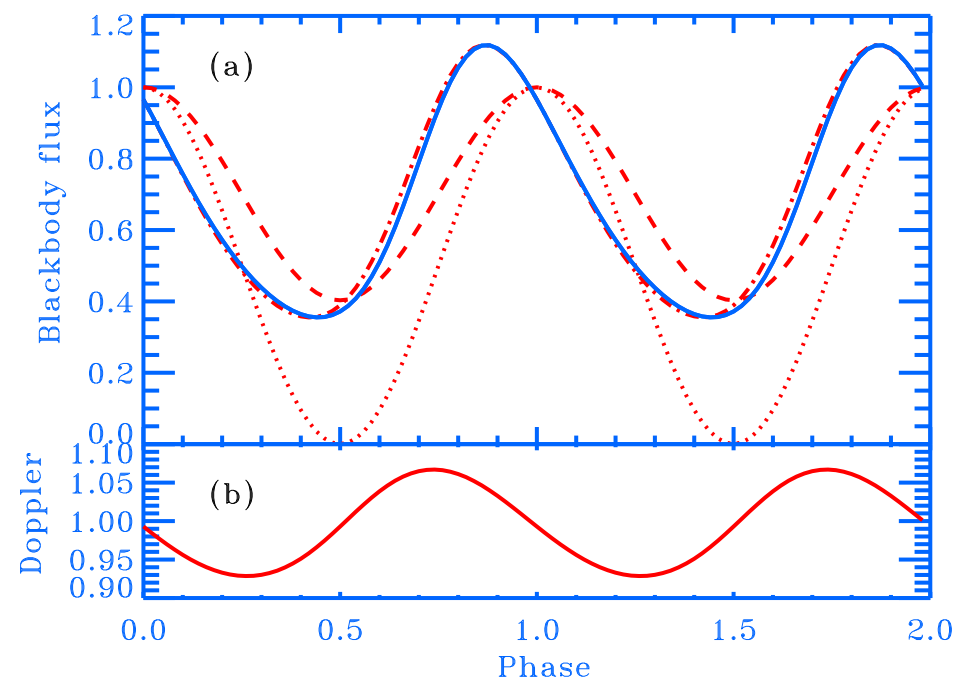

Fig. 5. Pulse profile for a small black body spot at the neutron star surface. (a) Dotted curve shows the profile for a slowly rotating star in Newtonian approximation. Gravitational light bending reduces variability amplitude (dashed curve). Doppler effect due to rapid rotation skews the profile (dot-dashed curves). Accounting for the light travel delays further modifies the profile slightly (solid curve). (b) Doppler factor as a function of phase. We assumed neutron star mass $M=1.4 \mathrm{M}_{\odot}$ and radius $R=10.3 \mathrm{~km}$, rotational frequency $\nu=600 \mathrm{~Hz}$, the inclination $i=45^{\circ}$, and the polar angle of the spot center $\theta=45^{\circ}$.

caused by the star's surface motion in the locally non-rotating frame. This was criticized by Braje et al. [5]. They also noticed that Doppler boost and light travel time delays are important for shaping the profiles, while the frame dragging affects them at $\mathrm{a} \sim 1 \%$ level. The Monte-Carlo simulations used in [5] for the light curve calculation are, however, extremely time-consuming and are hardly suitable for data fitting.

As frame dragging is not so important, one can consider Schwarzschild metric instead. One can account for Doppler effect making Lorentz transformation from the frame rotating with the star to a non-rotating frame and then follow light trajectory in the Schwarzschild space-time to infinity ("Schwarzschild + Doppler" or SD approach). Some attempt to formulate the problem was done in [35] who considered the spot and the observer in the equatorial plane only. Oscillation waveforms and amplitudes for arbitrary spot and observer position were computed in $[55,38]$. These paper provided no details on how to account for effect of aberration on intensity and projected spot area. A formalism for computing the pulse profiles accounting for Doppler boosting, relativistic aberration and gravitational bending appeared for the first time in [45]. Effects of the anisotropy of Comptonized radiation from a shock on the light curves and polarization were studied in [54]. Analytical formulae for oscillation amplitudes are presented in [43,44]. Light curves from realistic spots produced by accretion onto inclined magnetic dipole are computed in [23] using formalism of $[45,54,1]$. Accuracy of the SD approach is discussed in [6]. 


\subsection{Main effects}

Let us now briefly describe the main effects shaping the pulse profiles of AMSPs. Without general or special relativistic effects, a small black body spot would produce sinusoidal variations (with possible eclipses) due to a change of the projected area. Light bending reduces the variability amplitude (compare dotted and dashed curves in Fig. 5), while the pulse remains almost sinusoidal [1]. Relativistic aberration and Doppler boosting modify the observed flux for rapidly spinning star. The projected area is changed by the Doppler factor $\delta$ due to aberration, and the frequency-integrated specific intensity is multiplied by $\delta^{4}$ (see [45]). Since $\delta$ reaches the maximum a quarter of the period earlier than the projected area (Fig. 5b), the pulse becomes skewed to the left (see Fig. 5 and compare it to Fig. 2). Light travel time delays slightly modify the profile further (note, that time delays are already accounted for in the flux calculations by one of the Doppler factors).

\subsection{Oscillation amplitudes}

Due to the combined action of the Doppler effect and time delays, the pulse profile deviates from a simple sine wave. (We consider below the situation where the spot is always visible.) The relative amplitudes (in the bolometric signal) of the harmonic $A_{1}$ to that of the fundamental $A_{0}$ grows approximately linearly with the rotational frequency [43]:

$$
\frac{A_{1}}{A_{0}} \approx \frac{5}{2} \frac{2 \pi R}{c} \nu_{\text {spin }} \sin i \sin \theta=0.16 \frac{R}{10 \mathrm{~km}} \frac{\nu_{\text {spin }}}{300 \mathrm{~Hz}} \sin i \sin \theta,
$$

where $i$ is the inclination and $\theta$ is the polar angle of the spot center. Even a slight deviation from the isotropic emission (e.g. in the form $I(\alpha) \propto 1+b \cos \alpha$, where $\alpha$ is the angle from the spot normal and $b$ is the anisotropy parameter) leads to a high harmonic content $[45,43,54]$ :

$$
\frac{A_{1}}{A_{0}}=\frac{b\left(1-R_{\mathrm{s}} / R\right) / 2 \sin i \sin \theta}{1+2 b\left[R_{\mathrm{s}} / R+\left(1-R_{\mathrm{s}} / R\right) \cos i \cos \theta\right]},
$$

where $R_{\mathrm{s}}=2 G M / c^{2}$. The harmonic content $A_{1} / A_{0}$ as well as the total rms are proportional to $\sin i \sin \theta[1,45,43]$. Therefore, one expects harmonics to be relatively stronger when rms is larger which is exactly what is observed. Increasing the spot size leads to a reduction of the amplitude of the harmonic first and of the total rms later $[55,43]$.

If the spectrum has a sharp cutoff, the rms amplitude of the pulse at energies above the cutoff increases dramatically. The flux varies as $\propto \delta^{3+\Gamma}[45,54]$, where $\Gamma(E)=1-\mathrm{d} \ln F_{E} / \mathrm{d} \ln E$ is the energy-dependent photon spectral 


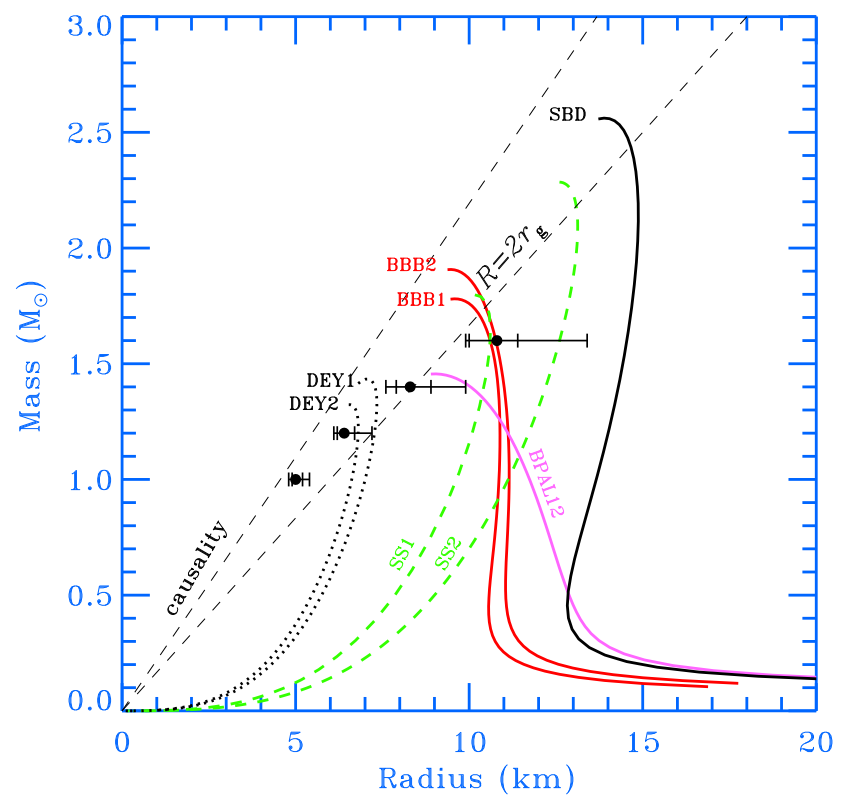

Fig. 6. Constraints on the neutron star mass-radius relation obtained by fitting the pulse profiles of SAX J1808.4-3658 (filled circles with error bars) together with a set of equations of state for neutron and strange stars. From [45].

index, resulting in the rms energy dependence $\propto 3+\Gamma(E)$. For the spectrum in the form $F_{E} \propto E^{-\left(\Gamma_{0}-1\right)} \exp \left(-\left[E / E_{\mathrm{c}}\right]^{\beta}\right)$, which is typical for Comptonization, the photon index $\Gamma(E)=\Gamma_{0}+\beta\left(E / E_{\mathrm{c}}\right)^{\beta}$. At low energies, $\Gamma \approx \Gamma_{0}$, and rms is a very weak function of energy. Close to the cutoff, the spectral index rapidly increases resulting in a significant grow of the rms, as observed in the INTEGRAL data for IGR J00291+5934 [13]. In case of coherent oscillations observed during X-ray bursts, a linear increase of the rms with energy is expected, since the spectrum is close to a blackbody which has an exponential cutoff (see also [35]).

\subsection{Constraints on the neutron star equation of state and spot parameters}

The amplitude of variability and the pulse shape (harmonic content) can be used to put constraints on the compactness of the neutron star, position, size and the emission pattern of a radiating spot. In case of the coherent oscillations observed during the X-ray bursts the constraints on the compactness are not very tight $[35,39,3]$. A very weak harmonic content led to the conclusion that the bright region (or the line of sight) must be near the rotational pole or must cover half of the star [38]. Fitting the pulse profile shape (but not the amplitude) of the oscillations observed during the X-ray burst of XTE J1814338 was performed in [3], who obtained the preferred spot polar angle $\theta=$ $90^{\circ} \pm 30^{\circ}$. This is highly improbable since an identical (in shape and phase) pulse observed in the persistent emission [52] would require the magnetic poles 
to lie at the equator and the antipodal spot should then be visible too.

For AMSPs the statistics in the pulse profiles is much better since the pulse is folded over a longer observational period (days or weeks rather than seconds as in the case of X-ray bursts). This allowed to obtain tight constraints on the neutron star compactness in SAX J1808.4-3658 as well as the emission pattern (consistent with Comptonization from the plane-parallel shock), inclination, and the spot polar angle [45] (see Fig. 6).

\section{Summary}

We have reviewed here X-ray observations of the AMSPs concentrating on the broad-band spectra and the energy-dependent pulse profiles. Recent advances in the theoretical modelling of the spectral and timing characteristics are also described. At the observational side, we are missing the details of the pulse profile and spectral evolution during the outbursts. One would expect that the changing accretion rate would force a change in the accretion pattern on to a neutron star surface, causing corresponding profile variations. Detailed studies of the reflection amplitude and frequencies of quasi-periodic oscillations should reveal changes in the inner disk radius during outbursts. The pulse profile should also react correspondingly as the visibility of the antipodal spot depends on the disk radius. Combined with the spin-up rate observations, these would shed some light on a complicated problem of the magnetosphere interaction with the accretion flow. On the theoretical side, we need a detailed model describing the dynamics of the accretion flow onto the neutron star surface including radiation feedback. It is not clear yet what fraction of the material follows the magnetic field lines towards the poles. The impact of the realistic spot geometry and the radiation pattern on the pulse profiles and the derived parameters should be studied further.

\section{References}

[1] Beloborodov A. M., 2002, ApJ, 566, L85

[2] Bhattacharya D., 1995, in Lewin W. H. G., van Paradijs J., van den Heuvel E. P. J., eds, X-ray Binaries, Cambridge University Press, Cambridge, p. 233

[3] Bhattacharyya S., Strohmayer T. E., Miller M. C., Markwardt C. B., 2005, ApJ, 619, 483

[4] Bildsten L., Chakrabarty D., 2001, ApJ, 557, 292

[5] Braje T. M., Romani R. W., Rauch K. P., 2000, 531, 447 
[6] Cadeau C., Leahy D. A., Morsink S. M., 2005, ApJ, 618, 451

[7] Chakrabarty D., Morgan E. H., 1998, Nature, 394, 346

[8] Chen K., Shaham J., 1989, ApJ, 339, 279

[9] Cui W., Morgan E. H., Titarchuk L. G., 1998, ApJ, 504, L27

[10] Cumming A., Zweibel E., Bildsten L., 2001, ApJ, 557, 958

[11] Eckert D., Walter R., Kretschmar P., et al. 2004, ATel 352

[12] Falanga M., Bonnet-Bidaud J. M., Poutanen J., et al. 2005, A\&A, 436, 647

[13] Falanga M., Kuiper L., Poutanen J., et al. 2005, A\&A, in press (astro-ph/0508613)

[14] Galloway D. K., Chakrabarty D., Morgan E. H., Remillard R. A., 2002, ApJ, 576, L137

[15] Galloway D. K., Markwardt C. B., Morgan E. H., Chakrabarty D., Strohmayer T. E., 2005, ApJ, 622, L45

[16] Gierliński M., Poutanen J., 2005, MNRAS, 359, 1261

[17] Gierliński M., Done C., Barret D., 2002, MNRAS, 331, 141

[18] Gilfanov M., Revnivtsev M., Sunyaev R., Churazov E., 1998, A\&A, 338, L83

[19] Haardt F., Maraschi L., 1993, ApJ, 413, 507

[20] Kaaret P., Morgan E., Vanderspek R., 2005, ATel 538

[21] Kirsch M. G. F., Mukerjee K. Breitfellner M. G., et al. 2004, A\&A, 423, L9

[22] Krauss M. I., Wang Z., Dullighan A., et al. 2005, ApJ, 627, 910

[23] Kulkarni A. K., Romanova M. M., 2005, ApJ, submitted (astro-ph/0507358)

[24] Leahy D. A., Li L., 1995, MNRAS, 277, 1177

[25] Malzac J., Beloborodov A. M., Poutanen J., 2001, MNRAS, 326, 417

[26] Markwardt C. B., 2004, in Kaaret P., Lamb F. K., Swank J. H., eds, X-ray Timing 2003: Rossi and Beyond. AIP, Melville, NY, p. 209

[27] Markwardt C. B., Swank J. H., 2002, IAUC, 7867, 1

[28] Markwardt C. B., Swank J. H., 2003, IAUC, 8144, 1

[29] Markwardt C. B., Swank J. H., Strohmayer T. E., in 't Zand J. J. M., Marshall F. E., 2002, ApJ, 575, L21

[30] Markwardt C. B., Smith E., Swank J. H., 2003, IAUC, 8080, 2

[31] Markwardt C. B., Swank J. H., Strohmayer T. E., 2003, ATel 164 
[32] Markwardt C. B., Galloway D. K., Chakrabarty D., Morgan E. H., Strohmayer T. E., 2004, ATel 360

[33] Markwardt C. B., Swank J. H., Strohmayer T. E., 2004, ATel 353

[34] Menna M. T., Burderi L., Stella L., Robba N., van der Klis M., 2003, ApJ, 589, 503

[35] Miller M. C., Lamb F. K., 1998, ApJ, 499, L37

[36] Morgan E. H., Chakrabarty D., Wijnands R., van der Klis M., Markwardt C. 2003, American Astronomical Society, HEAD meeting \#7, Abstract \#17.29

[37] Morgan E., Kaaret P., Vanderspek R., 2005, ATel 523

[38] Muno M. P., Özel F., Chakrabarty D., 2002, ApJ, 581, 550

[39] Nath N. R., Strohmayer T. E., Swank J. H., 2002, ApJ, 564, 353

[40] Nelson L. A., Rappaport S., 2003, ApJ, 598, 431

[41] Page D., 1995, ApJ, 442, 273

[42] Pechenick K. R., Ftaclas C., Cohen J. M., 1983, ApJ, 274, 846

[43] Poutanen J., 2004, in Kaaret P., Lamb F. K., Swank J. H., eds, X-ray Timing 2003: Rossi and Beyond. AIP, Melville, NY, p. 228 (astro-ph/0401209)

[44] Poutanen J., Beloborodov A. M., 2005, in preparation

[45] Poutanen J., Gierliński M., 2003, MNRAS, 343, 1301

[46] Poutanen J., Svensson R., 1996, ApJ, 470, 249

[47] Remillard R. A., Swank J. H., Strohmayer T. E., 2002, IAUC 7893, 1

[48] Riffert H., Mészáros P., 1988, ApJ, 325, 207

[49] Romanova M. M., Ustyugova G. V., Koldoba A. V., Lovelace R. V. E., 2004, ApJ, 610, 920

[50] Rupen M. P., Dhawan V., Mioduszewski A. J., 2004, ATel 364

[51] Strohmayer T., Bildsten L., 2003, in Lewin W. H. G, van der Klis M., eds, Compact Stellar X-Ray Sources. Cambridge University Press, Cambridge, in press (astro-ph/0301544)

[52] Strohmayer T. E., Markwardt C. B., Swank J. H., in 't Zand J., 2003, ApJ, 596, L67

[53] Vanderspek R., Morgan E., Crew G., et al. 2005, ATel 516

[54] Viironen K., Poutanen J., 2004, A\&A, 426, 985

[55] Weinberg N., Miller M. C., Lamb D. Q., 2001, ApJ, 546, 1098

[56] Wijnands R., 2005, in Pulsars New Research. Nova Science Publishers, New York, in press (astro-ph/0501264)

[57] Wijnands R., van der Klis M., 1998, Nature, 394, 344 